\title{
Beam Delivery for the Fermilab Mu2e Experiment
}

\section{Kevin R. Lynch*†}

Department of Earth and Physical Sciences, York College, Jamaica, NY, USA and The Graduate Center, The City University of New York, New York, NY, USA

E-mail: klyncheyork. cuny.edu

The Mu2e Experiment at Fermilab will search for the coherent, neutrinoless conversion of a muon to an electron in the field of an atomic nucleus. Such charged lepton flavor violating events have never been observed, but are predicted to occur in many Beyond the Standard Model scenarios at rates accessible to our experiment. I outline the key accelerator issues for the experiment, our progress on design and construction to date, and schedule for the coming years.

The 19th International Workshop on Neutrinos from Accelerators-NUFACT2017

25-30 September, 2017

Uppsala University, Uppsala, Sweden

* Speaker.

${ }^{\dagger}$ On behalf of the Mu2e Collaboration: http://mu2e. fnal.gov 


\section{Introduction}

The Mu2e effort holds a prominent place in the near term future of the U.S. High Energy Physics program. In fact, the recent report of the Particle Physics Project Prioritization Panel (P5) which advises the U.S. Government on HEP community priorities - advises completion of the Mu2e Experiment under all budget scenarios considered [1]. With a project baseline cost of $\$ 270$ million, this involves a significant investment of available resources; why, then, this level of interest?

Although charged lepton flavor violation (CLFV) has never been observed experimentally, we know that it must occur: neutrino flavor oscillations coupled with loops guarantees the existence of CLFV; see Figure 1. However, even with the most optimistic parameter values in the PMNS neutrino mixing matrix $U$, the Standard Model rate prediction is tiny

$$
\operatorname{Br}(\mu \rightarrow \mathrm{e} \gamma)=\frac{3 \alpha}{32 \pi}\left|\sum_{k=2,3} U_{\mu k}^{*} U_{\mathrm{e} k} \frac{\Delta m_{1 k}^{2}}{M_{\mathrm{W}}^{2}}\right|^{2}<10^{-54} .
$$

There is no conceivable experiment which could observe a branching ratio this small. While this initially seems disappointing, it is a major opportunity in disguise as any experimental observation of CLFV becomes incontrovertible evidence for new physics Beyond the Standard Model (BSM)!

Searches for CLFV have a long and distinguished history of guiding both theorists and experimentalists in elucidating the foundations of particle physics. For at least seventy years, there has been a long line of experiments searching for violations in both meson and lepton decays. Early non-observation of $\mu \rightarrow \mathrm{e} \gamma$ at the $10 \%$ level laid rest to the notion that the muon was simply an electromagnetic excitation of the electron [2]. Later, the non-observation of the same signal at the $10^{-8}$ level proved that muon and electron neutrinos were distinct species [3].

Today, CLFV searches form their own cottage industry: there are numerous ongoing searches in many meson and tau channels at the LHC, while there are a number of significant efforts worldwide in muon decays. A large number of these efforts were represented at this workshop. In $\mu \rightarrow \mathrm{e} \gamma$, the MEG search at PSI has pushed down nearly to the limits of their sensitivity [4], while the Mu3e developments at PSI promise a very sensitive search for $\mu^{+} \rightarrow \mathrm{e}^{+} \mathrm{e}^{+} \mathrm{e}^{-}$[5]. Neutrinoless conversion provides perhaps the most promising avenue for large sensitivity improvements, with at least three ongoing efforts at advanced stages of development: DeeMee [6] and COMET [7] at the JPARC, and the Mu2e Experiment at Fermilab [8]. Both Mu2e and COMET envision improvement over the current bounds by four orders of magnitude compared to the SINDRUM-II experiment [9],

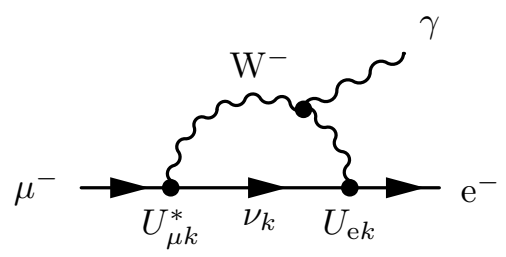

Figure 1: A Standard Model source of charged lepton flavor violating $\mu \rightarrow \mathrm{e} \gamma$ arises from neutrino flavor oscillations within loops; these events have an unmeasurably small branching ratio. 
giving access to energy scales as high as $10^{4} \mathrm{TeV}$, well in excess of processes directly accessible at the LHC.

Conversion experiments have significantly higher reach than other channels, because they have a major kinematic advantage. In, say, muon to electron conversion, the signal electrons of interest are hard to distinguish from the bulk of electrons from the vast background of Michel electrons from normal muon decay. In contrast, because the conversion signal comes from the two body decay of a heavy muonic atom, the signal electrons in the conversion process are monochromatic with an energy roughly that of the muon mass. This puts the signal well above the vast bulk of the background, and just beyond the high energy recoil tail from normal muon decay in orbit (DIO). Herein lies the advantage of the conversion channel, and the experiments are designed to take advantage of this kinematic separation.

For more details of other CLFV searches, please see the contribution by R. Bernstein to this workshop.

\section{The Design of Mu2e}

The Mu2e Experiment is under construction at the Fermi National Accelerator Laboratory in Batavia, Illinois in the United States. Along with the Fermilab Muon $g-2$ Experiment, Mu2e will occupy the new Muon Campus facility close to Wilson hall, forming the core of a muon program for at least the next decade.

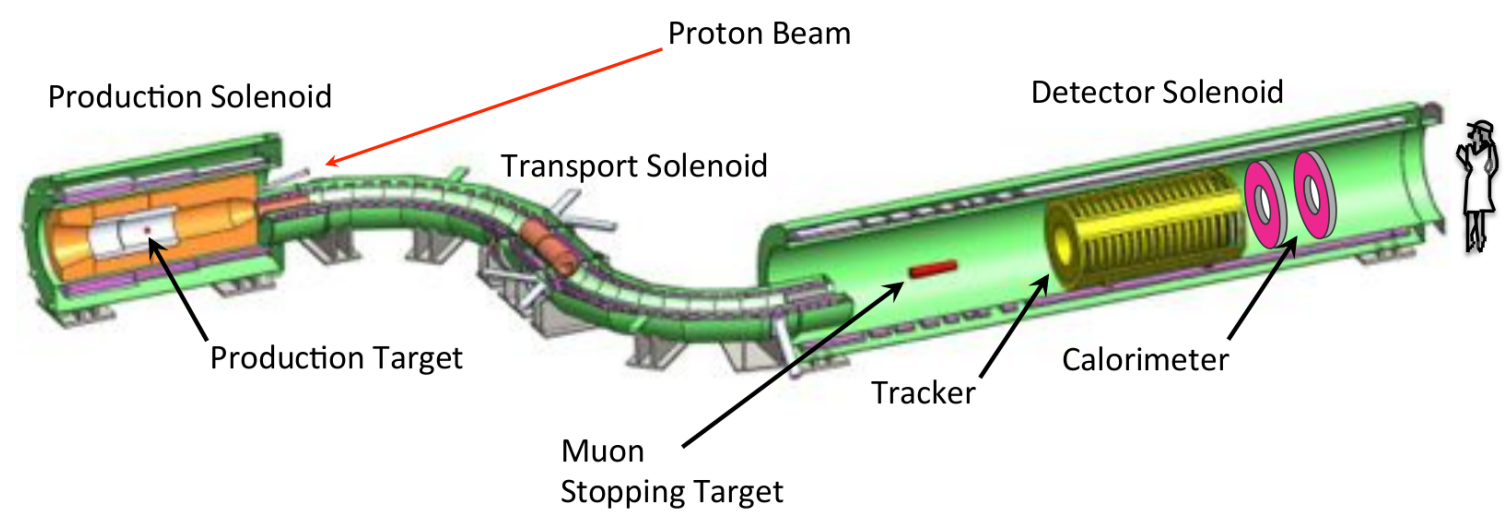

Figure 2: The Mu2e Apparatus, showing cutaways of the three solenoids.

The Mu2e apparatus will separate the production of muons from observation of their decays; see Figure 2. Muons will be produced within the Production Solenoid (PS) and their decays will be observed by a suite of detectors within the Detector Solenoid (DS). An "S"-shaped Transport Solenoid (TS) will be responsible for muon beam transport between the other solenoids.

The PS produces a backward moving muon beam to dramatically reduce beam related backgrounds. The proton beam enters slightly off axis, in the gap between the PS and TS. The production target is a pencil-sized tungsten rod, held in place by a "bicycle wheel" support system, and cooled by direct radiation to the vacuum. The stainless steel vacuum vessel holding the production target is surrounded by a bronze and water heat and radiation shield to protect the PS superconducting coils from both heat load and radiation damage. The PS endcap provides windows for 
passing the spent beam to a downstream proton beam stop, along with a maintenance window to allow for target changes. Because of the heavy activation of the target and PS, target changes will be performed by a robotic remote handling system. The field in the PS is graded from $4.5 \mathrm{~T}$ at the proton-downstream end to $2.5 \mathrm{~T}$ at the entrance to the TS. This graded field acts both as a mirror increasing muon acceptance, as well as sweeping particles towards the TS, to prevent long-lived storage of secondaries within the PS that could later escape and arrive in the DS during the live window.

The "S"-shaped solenoid TS sweeps muons from the PS to the DS. The field is also graded inside TS, again to ensure that particles do not become trapped in long-lived orbits. The solenoid is curved to ensure that there is no line-of-sight path between the production target and the detectors, to reduce contamination by neutral particles. The entrance to and exit from the TS are occupied by collimators to define the acceptance of the channel. The most interesting aspect of this design is the effect a curved solenoid has on charged particle trajectories: charged particles drift in the non-bend direction (vertically in our case), with particles of opposite sign drifting in opposite directions. This naturally charge separates the beam in the vertical direction. We insert an asymmetric collimator in the central straight section to sign select on negative muons. The recurved section past the central collimator moves the beam back on-axis at the entrance to the DS.

The Detector Solenoid forms the heart of the experiment. As the beam enters the DS, it first encounters a series of aluminum stopping target foils. These are mounted within a graded magnetic field region; again, the grade reflects decay products towards the downstream end of the DS, increasing the acceptance of the detector systems. Beyond the stopping target, the field becomes uniform over the detector train. The primary measurement device is a low-mass, straw-tube electron tracker; with a wall thickness of only $15 \mu \mathrm{m}$, the tracker has a high-side resolution of less than $180 \mathrm{keV}$, thanks to the low mass design. The dynamic range issues are solved by simply not measuring electrons in the peak of the DIO distribution: the tracker has a central hole along its axis that passes particles below $55 \mathrm{MeV} / \mathrm{c}$ without measurement. Following the tracker is a scintillating crystal calorimeter, made from two annular disks. The calorimeter provides a redundant energy measurement to the tracker, as well as powerful particle ID capability, and independent trigger and track seeding capabilities. The final major component mounted within the DS is a muon beam stop, which intercepts and absorbs the beam particles that are not stopped by the target foils, and decay products below measurement threshold. A four layer scintillating plastic Cosmic Ray Veto system surrounds the top and sides of the DS to virtually eliminate cosmic ray muons that could fake conversion signals; to reduce the rate from one per day to less than 0.1 event during the three year duration of the experiment, this system must operate at a $99.99 \%$ detection efficiency.

While the bulk of the Mu2e experiment is designed for the detection of conversion events, that's only half the equation (literally!). To measure the conversion rate (or branching ratio), we have to normalize the number of conversion candidates to some proxy for the total number of muon stops. This is the job of the final detector, the Stopping Target Monitor (STM). The baseline design is for a High Purity Germanium detector that will view through very small solid angle the stopping target foils, and will count the characteristic atomic cascade transition x-rays. Given acceptance and efficiency measures for both the conversion event counting and the cascade $\mathrm{x}$-ray counting, we can determine the ratio of conversions to nuclear capture events; our final result will be the 
conversion ratio

$$
R_{\mu \mathrm{e}}=\frac{\mu^{-}+\mathrm{A}(Z, N) \rightarrow \mathrm{e}^{-}+\mathrm{A}(Z, N)}{\mu^{-}+\mathrm{A}(Z, N) \rightarrow v_{\mu}+\mathrm{A}(Z-1, N)}
$$

For a three year run, we expect less than half a background event in the signal window; our goal for single event sensitivity is $2.5 \times 10^{-17}$, a four order of magnitude improvement over the SINDRUM II result. For a conversion ration $R_{\mu \mathrm{e}} \sim 10^{-15}$, we will see fifty conversion events during the run.

For more details on the design and status of Mu2e, please see the contribution by C. Dukes to this workshop.

\section{Proton Beam Delivery}

To achieve such a low background event rate - primarily prompt pion capture events - requires a pulsed primary beam. In this scheme, protons arrive at a production target in pulses, producing bunches of pions. These pions are both transported toward the stopping target, as well as decaying to muons. Those pions arriving at the stopping target - like their muon counterparts - will undergo atomic and nuclear capture, producing fluxes of secondaries that can mimic the conversion signal. Given the much shorter lifetime of the pion compared to the muon, this major background can eliminated by waiting out their decays. To optimize data collection, the next proton pulse should arrive at the production target roughly two muon lifetimes after the previous pulse; the negative muon lifetime in Aluminum is about $864 \mathrm{~ns}$.

The Fermilab proton acceleration chain begins with an $\mathrm{H}^{-}$linac which feeds $400 \mathrm{MeV}$ beam to the Booster synchrotron; the beam is foil stripped to protons during injection into the Booster. The Booster then raises the kinetic energy of the protons to the $8 \mathrm{GeV}$ used by the rest of the complex. For Mu2e, proton batches will be extracted into the MI- 8 beamline and transferred to the permanent magnet Recycler Ring. In the Recycler, RF manipulation of the circulating protons will rebunch each batch into four bunches of approximately $100 \mathrm{~ns}$ duration. One bunch at a time will be fast extracted from the Recycler to the Delivery Ring (part of the former anti-proton source). This stored beam will be slow extracted at a rate of $4 \times 10^{7}$ protons per orbit into the new M4 beamline constructed for Mu2e. The Delivery Ring has a nearly ideal cyclotron period for Mu2e: $1695 \mathrm{~ns}$, almost exactly twice the lifetime of negative muons in Aluminum. The final beam will arrive at the production target inside the Mu2e PS with a nearly Gaussian, $1 \mathrm{~mm} \times 1 \mathrm{~mm}$ profile. The target itself is a radiatively cooled tungsten rod, $160 \mathrm{~mm}$ in length and $6.3 \mathrm{~mm}$ in diameter, suspended within the PS vacuum volume by a "bicycle wheel" hub-and-spoke system. The spent beam will exit through a window on the back end of the PS, and be absorbed by an air cooled, steel core beam absorber downstream of the PS.

There are a number of novel developments required for successful operations of this beam transport system, particularly related to spill regulation and extinction of out-of-time beam. Slow extraction of beam from the Delivery Ring requires careful production and control of a large beam instability within the Ring. The quadrupoles within the Ring will be ramped to drive a $1 / 3$ integer resonance in the horizontal tune. Sextupole magnets will be used to drive the controlled beam instability, causing beam growth in the horizontal plane. An electrostatic septum will peel off a 
microbunch during each orbit. A spill monitor and dynamic feedback system will control an RF Knockout system to control the spill intensity. The useful beam will be extracted over roughly 32,000 turns, with residual beam kicked to an abort line. This scheme will result in spill-to-spill variations no larger than $50 \%$ of the nominal intensity.

While the pulsed beam is necessary for successful operation of the experiment from a background reduction perspective, it is not sufficient: any protons arriving at the production target outside of the nominal $100 \mathrm{~ns}$ window will produce out-of-time pions that do not have the opportunity to decay before the measurement window opens. Out-of-time arrivals must be suppressed to below one part in $10^{10}$ in order for us to reach our single event sensitivity goal. A very small fraction of this suppression comes from the slow extraction control from the Delivery Ring (about $2 \times 10^{-5}$ ). The bulk of the required suppression will come from an active AC dipole magnet system in the M4 line. The AC dipole system will consist of upstream tail and halo collimators to clean the beam, followed by the dipole itself, followed by an extinction collimator. The dipole excitation will consist of two superposed harmonics: one large amplitude at $300 \mathrm{kHz}$ to give a large kick to the beam, and a smaller amplitude at $4.5 \mathrm{MHz}$ to minimize the field in the neighborhood of the zero crossings of the lower frequency harmonic. These zero crossings will be timed to the passage of the extracted beam pulses. Total extinction is expected to be of order $10^{-12}$, comfortably in excess of the requirement.

\section{Accelerator Status and Schedule}

The Fermilab Muon Campus commenced operations in Spring of 2017 for the Muon $g-2$ experiment. Mu2e and $g-2$ both use the Delivery Ring, and share space in the beamline enclosure downstream of the extraction region ${ }^{1}$; the magnets in this section of the M4 line have been installed and aligned. Construction continues on the rest of the line, assisted by the installation of a temporary shielding labyrinth and interlocked gates; further installation of components upstream of the labyrinth must occur during scheduled accelerator complex maintenance shutdowns.

Installation of magnets and other components will continue in this configuration until the Summer of 2020; during this shutdown period, the shield wall will be moved to just upstream of the Production Target hall, allowing the beamline to be fully commissioned using single turn fast extraction of protons from the Delivery Ring, through the M4 line, to a diagnostic absorber. Resonant extraction will be commissioned through the diagnostic absorber early the following year. We expect first delivery of low intensity beam to the production target late in 2021, followed by final tuning and at least three years of experiment operations.

\section{Future Prospects}

As mentioned earlier, because the Standard Model does not predict observable levels of CLFV, the value of Mu2e is high whether or not we see a signal. In either case, a future extension of the

\footnotetext{
${ }^{1}$ The Delivery Ring is used for proton storage and delivery by Mu2e, but as a pion decay line for Muon $g-2$; operation of both experiments in parallel is not possible, as various devices must be swapped out to change operating modes.
} 
experiment - Mu2e-II - is under active study. The proposed Deep Underground Neutrino Experiment (DUNE) will not be possible without a substantial increase in the beam power provided by the Fermilab accelerator complex. The main accelerator upgrade to enable the multi-megawatt Long Baseline Neutrino Facility (LBNF) plan is a replacement of the aging Linac frontend with a high power, superconducting, CW capable frontend. This frontend - dubbed PIP-II, for Proton Improvement Plan II - will be able to deliver significantly more beam than required for LBNF operation. Mu2e-II is envisioned to directly utilize $100 \mathrm{~kW}$ of the available power, as well as the configurable pulse structure from the new machine. This will require the construction of a new beamline enclosure, and the upgrade of many components of the experiment. We believe an additional factor of 10 improvement in single event sensitivity is possible without major changes to the fundamental concept of the experiment.

If we do see a signal in our first run, an upgraded experiment using multiple different target materials could help elucidate the physics sources responsible for CLFV. If we do not see a signal in our first run, an upgrade will allow us to improve our sensitivity and probe higher energy scales. In either case, the result from Mu2e combined with other CLFV experiments, results from Muon $g-2$, and direct searches at the LHC will help point us in the right direction to define the next Standard Model.

\section{Acknowledgments}

We are grateful for the vital contributions of the Fermilab staff and the technical staff of the participating institutions. This work was supported by the US Department of Energy; the Italian Istituto Nazionale di Fisica Nucleare; the Science and Technology Facilities Council, UK; the Ministry of Education and Science of the Russian Federation; the US National Science Foundation; the Thousand Talents Plan of China; the Helmholtz Association of Germany; and the EU Horizon 2020 Research and Innovation Program under the Marie Sklodowska-Curie Grant Agreement No.690385. Fermilab is operated by Fermi Research Alliance, LLC under Contract No. De-AC02-07CH11359 with the US Department of Energy, Office of Science, Office of High Energy Physics. The United States Government retains and the publisher, by accepting the article for publication, acknowledges that the United States Government retains a non-exclusive, paid-up, irrevocable, world-wide license to publish or reproduce the published form of this manuscript, or allow others to do so, for United States Government purposes.

\section{References}

[1] Particle Physics Project Prioritization Panel (P5), Building for Discovery: Strategic Plan for U.S. Particle Physics in the Global Context, .

[2] E. P. Hincks and B. Pontecorvo, Search for gamma-radiation in the 2.2-microsecond meson decay process, Phys. Rev. 73 (1948) 257-258.

[3] G. Danby et al., Observation of High-Energy Neutrino Reactions and the Existence of Two Kinds of Neutrinos, Phys. Rev. Lett. 9 (1962) 36-44.

[4] MEG collaboration, J. Adam et al., New constraint on the existence of the $\mu^{+} \rightarrow e^{+} \gamma$ decay, Phys. Rev. Lett. 110 (2013) 201801, [1303.0754]. 
[5] Mu3E collaboration, N. Berger, The Mu3e Experiment, Nucl. Phys. Proc. Suppl. 248-250 (2014) $35-40$.

[6] DEEME collaboration, M. Aoki, A new idea for an experimental search for nu-e conversion, PoS ICHEP2010 (2010) 279.

[7] COMET collaboration, Y. G. Cui et al., Conceptual design report for experimental search for lepton flavor violating mu- - e-conversion at sensitivity of $10^{-16}$ with a slow-extracted bunched proton beam (COMET), KEK-2009-10 (2009) .

[8] Mu2E collaboration, L. Bartoszek et al., Mu2e Technical Design Report, FERMILAB-TM-2594, FERMILAB-DESIGN-2014-01 (2014), [1501.05241].

[9] SINDRUM II collaboration, C. Dohmen et al., Test of lepton flavor conservation in mu $\rightarrow e$ conversion on titanium, Phys. Lett. B317 (1993) 631-636. 